ORIGINAL ARTICLE

\title{
Clinical examination of athletes with groin pain: an intraobserver and interobserver reliability study
}

\author{
P Hölmich, L R Hölmich, A M Bjerg
}

Br J Sports Med 2004;38:446-451. doi: 10.1136/bjsm.2003.004754

See end of article for authors' affiliations

.....................

Correspondence to: Associate Professor P Hölmich, Amager University Hospital, Orthopaedic Surgery Department, Italiensvej 1 Copenhagen DK-2300 S, Denmark; per.holmich@ ah.hosp.dk

Accepted 26 March 2003
Background: Groin pain is a diagnostic and therapeutic challenge to sports medicine. The literature provides no consensus on definitions of or diagnostic criteria for groin pain in athletes. To compare the results of research and treatments, the methods used to diagnose and evaluate the degree of groin pain must be clearly defined and reproducible.

Objectives: To describe clinical examination techniques for groin pain in athletes and evaluate the intraobserver and interobserver reliability of these.

Methods: Eighteen athletes, nine with sports related groin pain and nine without groin pain, were examined by two doctors and two physiotherapists. The examiners were trained in the examination techniques before the study. The examiners were blinded to the symptoms and identity of the subjects. The subjects were examined twice by each examiner in random order. The examinations included evaluation of adductor muscle related pain and strength, iliopsoas muscle related pain, strength, and flexibility, abdominal muscle related pain, and strength and pain at the symphysis joint. Kappa statistics and percentage of agreement were used to evaluate the data.

Results: Overall, the $\kappa$ values and percentage of agreement were in accordance and showed good reliability of the examinations. The $\kappa$ values for the intraobserver agreement were above 0.60 in 11 of 14 tests, and those for the interobserver agreement of the pain tests were above 0.60 in eight of 10 tests. The only test without acceptable interobserver reliability was the strength test for iliopsoas muscle.

Conclusion: All but one of the tests investigated were reproducible and subject only to limited intraobserver and interobserver variation.
G roin pain is associated with many sports and represents a diagnostic and therapeutic challenge. ${ }^{12}$ In soccer, the incidence of groin injury has been estimated to be $10-18$ per 100 soccer players per year. ${ }^{3-5}$ However, the definitions of and diagnostic criteria for groin pain in athletes are not clear, and in the literature no consensus is provided. To compare the results of research and treatments, the tools used to diagnose and evaluate the degree of groin injury must be clearly defined and reproducible.

Techniques to evaluate pain related to, and the strength and flexibility of, the adductor muscles, iliopsoas muscle, and abdominal muscles, and pain at the symphysis joint are in our experience important tools in the clinical examination of athletes with groin pain. ${ }^{26}$ There is no reference in the literature on how these tests should be performed.

The purpose of this study was to evaluate the intraobserver and interobserver variation in the results of standardised clinical examination techniques for groin pain in athletes.

\section{MATERIAL AND METHODS}

A standardised clinical groin examination was carried out by two doctors and two physiotherapists, all experienced clinicians. One doctor $(\mathrm{PH})$ trained the other examiners in the techniques, and a pilot study was conducted to correct individual examiner's use of the techniques.

Eighteen young adult male subjects were tested. All were athletes, primarily soccer players. Nine had long standing (more than three months) sports related groin pain affecting their adductor muscle(s), iliopsoas muscle(s), and/or symphysis joint. The remaining nine had no recent (more than three years) history of groin pain of any kind. Three examiners tested all 18 subjects, but the fourth examiner was unavailable for examination of six subjects. The examinations were performed in three sessions with six subjects participating in each, three of the subjects in each session being patients with groin pain.

The examiners were blinded to the identity of the subjects. The subjects were placed supine on examination couches with a curtain hanging down over the abdomen leaving only the legs and pelvis visible to the examiner. All subjects were wearing the same type of hospital underwear. When the examination required the examiner to place his hands above the pelvis (the abdominal tests: 6A, B, and C (appendix 1)), this was done behind the curtain so that the identity of the subject was not revealed.

The subjects were examined in random order, four at a time. The examiners left the room between each examination, and a secretary replaced the subjects at random for repeated examination. When all examiners had examined all subjects once, the subjects were examined again in the same setting, but this time in a different random order. A secretary recorded the results of the examinations on a standardised data sheet.

The study was approved by the local ethics committee.

The examination techniques tested were:

1. Adduction of the legs against resistance; pain and strength were evaluated.

2. Palpation of the insertion of the adductor longus muscle at the pubic bone; pain was evaluated.

3. Passive stretching of the adductor muscles; pain was evaluated.

4. Palpation of the symphysis joint; pain was evaluated.

5. Palpation of the rectus abdominis muscle at the pubic bone; pain was evaluated 
6. Functional testing of the abdominal muscles; pain and strength were evaluated.

7. Palpation of the psoas muscle above the inguinal ligament; pain was evaluated.

8. Functional iliopsoas test; pain and strength were evaluated.

9. Passive stretching of the iliopsoas muscle (the Thomas' test modified $^{7}$ ); pain and tightness were evaluated.

The details of the examination techniques are described in the appendix.

\section{Statistical methods}

To determine the degree of agreement within and between the observers, we used percentage of agreement, which is a simple calculation of the number of tests with agreement against the total number of tests performed, and kappa statistics, which takes into account the agreement expected solely on the basis of chance. $\kappa$ values of $0.41-0.60$ indicate moderate agreement, $0.61-0.80$ good agreement, and 0.811.00 very good agreement. ${ }^{8}$ To determine the $\kappa$ value for the interobserver agreement between four observers, the method suggested by Siegel and Castellan ${ }^{9}$ was used. The value for percentage of agreement for the interobserver agreement is calculated as the mean of the six values for agreement between the four observers. In some of the tests, the structures to be tested were paired, and two similar tests were performed, one on the right and one on the left side. As the side tested was not the subject of this reliability study, a mean value of the $\kappa$ values and the percentages of agreement of the two sides was calculated as the final result of each test.

\section{RESULTS}

\section{Intraobserver reliability}

Overall, the $\kappa$ values and percentages of agreement were in good accordance. The $\kappa$ values were above 0.60 in 11 of the 14 tests and above 0.80 in six tests. In three tests, the values were below 0.60 (table 1). The percentage of agreement ranged from 85.4 to 96.5 (table 1). In three tests, there was discrepancy between the $\kappa$ values and the percentage of agreement: $(a)$ the $\kappa$ value of the psoas functional pain test (8A) was 0.31 , but the percentage of agreement was $90.3 ;(b)$ the $\kappa$ value of the abdominal strength test $(6 \mathrm{~B})$ was -0.03 , but the percentage of agreement was $94.4 ;(c)$ the $\kappa$ value of the abdominal oblique functional pain test $(6 \mathrm{C})$ was 0.51 , but the percentage of agreement was 91.0.

\section{The interobserver reliability}

The tests for interobserver reliability showed overall good agreement between the four observers. In the tests for pain, the interobserver $\kappa$ values were above 0.60 in eight tests and above 0.80 in five. In two tests, the values were below 0.60 . The percentages of agreement were above 80 in 10 pain tests and above 90 in eight pain tests (table 2). In two pain tests, there were discrepancies between the $\kappa$ values and the percentage of agreement: $(a)$ the $\kappa$ value of the abdominal oblique functional pain test (6C) was 0.41 whereas the percentage of agreement was $87.0 ;(b)$ the $\kappa$ value for the abdominal functional pain test $(6 \mathrm{~A})$ was 0.57 whereas the percentage of agreement was 90.3. The only test that had both a low $\kappa$ value and a low percentage of agreement was the iliopsoas strength test $(8 \mathrm{~B})$. The other tests for strength and flexibility had $\kappa$ values of $0.05-0.29$; in contrast with these low values, the percentages of agreement for the same tests were 83.2-92.6.

\section{DISCUSSION}

Most of the tests, in both the intraobserver and interobserver reliability study, found a "good" or "very good" degree of agreement. ${ }^{8}$ The disagreement between the $\kappa$ values and the percentage of agreement for certain of the tests was probably the result of a skewed distribution of the marginals, a problem to which $\kappa$ is very sensitive. ${ }^{8}$ For the interpretation of the results in these tests, we have used the percentage of agreement.

The intraobserver agreement in this study was good because all tests but one had a percentage of agreement over 90 , and all but three tests had a $\kappa$ value exceeding 0.60 .

The interobserver agreement of the iliopsoas strength test (8B) showed it to be the only test in this study not reproducible on an acceptable level. As performed in this study, it requires a fairly powerful pull on the leg by the examiner, and is thus susceptible to the individual strength of the examiner. The interobserver agreement in the tests on pain was good. The only two tests with $\kappa$ values below 0.60 were both over 0.40 and had a satisfactory percentage of agreement.

The very high percentages of agreement as well as $\kappa$ values for the adductor muscle and iliopsoas muscle tests on pain (tests 1A, 2A, 3A, and 9B) are important results. These muscles play an essential part in the cause, diagnosis, and treatment of groin pain in athletes, ${ }^{126}$ and it is very important to have good and reproducible techniques to examine both pain and function.

\begin{tabular}{|c|c|c|c|c|c|c|}
\hline \multirow[b]{2}{*}{ Test } & \multicolumn{3}{|c|}{ Percentage of agreement } & \multicolumn{3}{|c|}{$\kappa$ value } \\
\hline & Right & Left & Mean & Right & Left & Mean \\
\hline Adductor functional pain (1A) & 95.8 & 97.2 & 96.5 & 0.91 & 0.91 & 0.91 \\
\hline Adductor palpation pain $(2 \mathrm{~A})$ & 95.8 & 94.4 & 95.1 & 0.88 & 0.91 & 0.89 \\
\hline Adductor stretching pain $(3 \mathrm{~A})$ & 94.4 & 93.1 & 93.8 & 0.66 & 0.68 & 0.67 \\
\hline Symphysis palpation pain (4A) & & & 93.1 & & & 0.84 \\
\hline Rectus abdominis palpation pain (5A) & 94.4 & 90.3 & 92.4 & 0.75 & 0.86 & 0.81 \\
\hline Abdominal functional pain (6A) & & & 93.1 & & & 0.63 \\
\hline Abdominal oblique functional pain $(6 \mathrm{C})$ & 88.9 & 93.1 & 91.0 & 0.58 & 0.44 & 0.51 \\
\hline Psoas palpation pain (7A) & 94.4 & 93.1 & 93.8 & 0.81 & 0.87 & 0.84 \\
\hline Psoas functional pain $(8 \mathrm{~A})$ & 87.5 & 93.1 & 90.3 & 0.52 & 0.11 & 0.32 \\
\hline Psoas stretching pain (9B) & 94.4 & 97.2 & 95.8 & 0.91 & 0.72 & 0.81 \\
\hline Adductor strength (1B) & 93.1 & 93.1 & 93.1 & 0.58 & 0.72 & 0.65 \\
\hline Abdominal strength (6B) & & & 94.4 & & & -0.03 \\
\hline Psoas strength (8B) & 83.3 & 87.5 & 85.4 & 0.64 & 0.59 & 0.61 \\
\hline Psoas flexibility (9A) & 90.3 & 94.4 & 92.4 & 0.83 & 0.66 & 0.74 \\
\hline
\end{tabular}

NB There are no values for left and right for symphysis palpation pain (4A), abdominal functional pain $(6 \mathrm{~A})$, or abdominal strength (6B). 


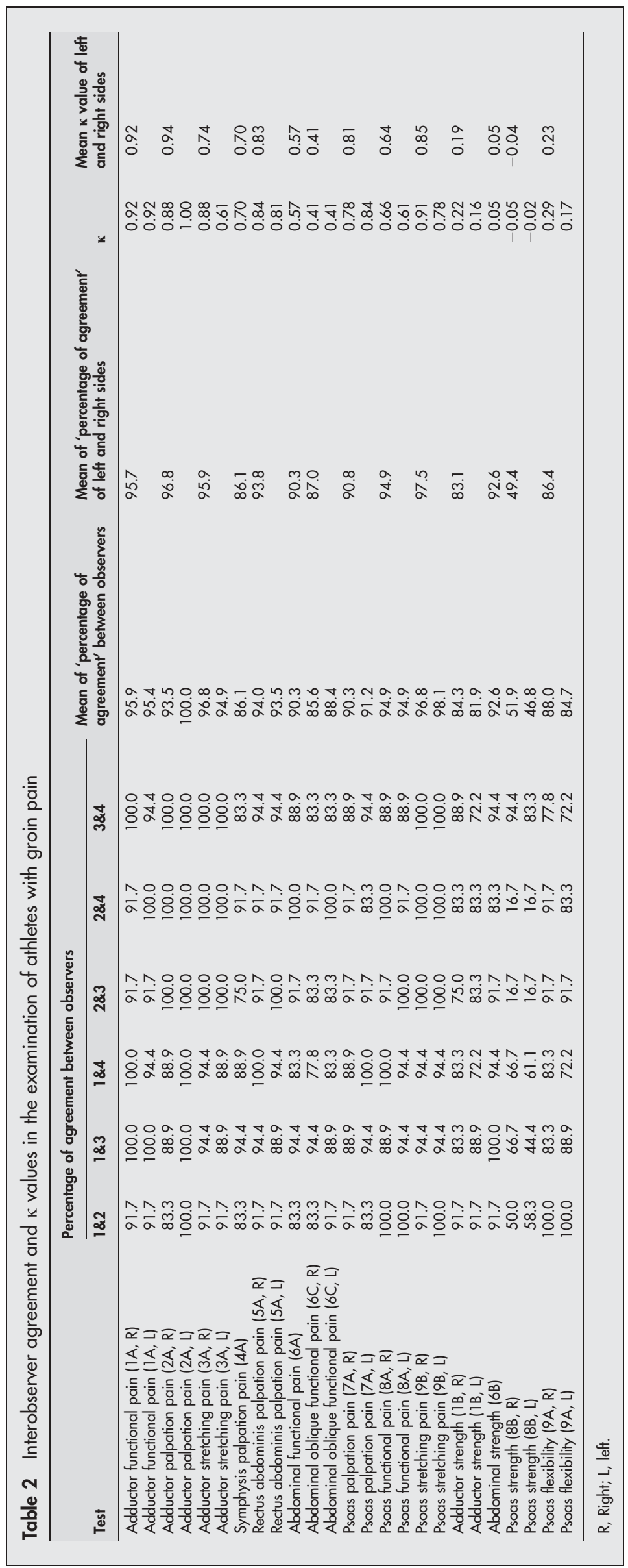


Generally, manual techniques have to be practised to be mastered. During the pilot study it became clear that training to perform the techniques was very important. None of the techniques are technically demanding, but the details, as described in appendix 1 , have to be learned in order to provide a meaningful basis for clinical use as well as for scientific evaluation.

To our knowledge, no previous studies have described these clinical examination techniques for groin pain in athletes and evaluated their reliability. Our study design effectively blinded the examiners to the identity of the subjects, which reduced the risk of bias to a minimum.

This study shows that all but one of the tests for pain, strength, and flexibility of the adductor muscles, the iliopsoas muscles, the abdominal muscles, and the symphysis joint are reproducible and are subject only to limited intraobserver and interobserver variation.

\section{ACKNOWLEDGEMENTS}

We thank Niels Bo Schmidt and Adam von Magius for help with the figures.

\section{Authors' affiliations}

P Hölmich, L R Hölmich, Amager University Hospital, Copenhagen, Denmark

A M Bjerg, Institute of Preventive Medicine, Copenhagen

\section{APPENDIX}

\section{EXAMINATION TECHNIQUES FOR THE EVALUATION OF GROIN PAIN IN ATHLETES USED IN THE INTRAOBSERVER AND INTEROBSERVER RELIABILITY STUDY}

Before the examinations, the subjects were told that the natural soreness or discomfort elicited by some of the tests should not be registered as pain.

\section{Adduction of the legs against resistance (fig l)}

The subject lies supine. The examiner stands at the end of the couch with hands and lower arms between the feet of the subject to hold them apart. The feet of the subject point straight up, and the subject presses them together with maximal force without lifting the legs or pelvis.

(A) The presence of pain in the groin is evaluated by the subject as "yes" or "no".

(B) The strength is evaluated by the examiner as "strong", "intermediate", or "weak".

\section{Palpation of the adductor longus muscle insertion (fig 2 )}

The subject lies supine. The leg to be tested is placed on a pillow with the hip flexed, abducted, and externally rotated,

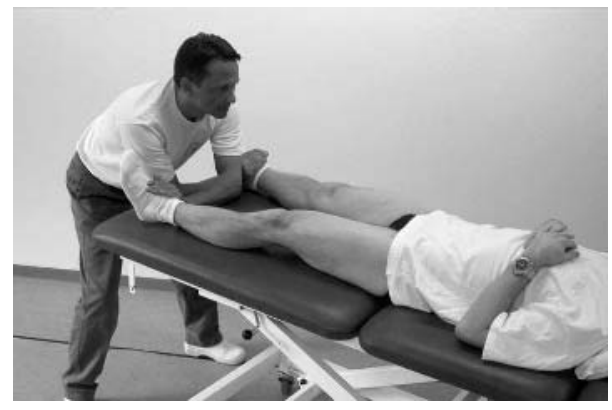

Figure 1 Adduction against resistance.

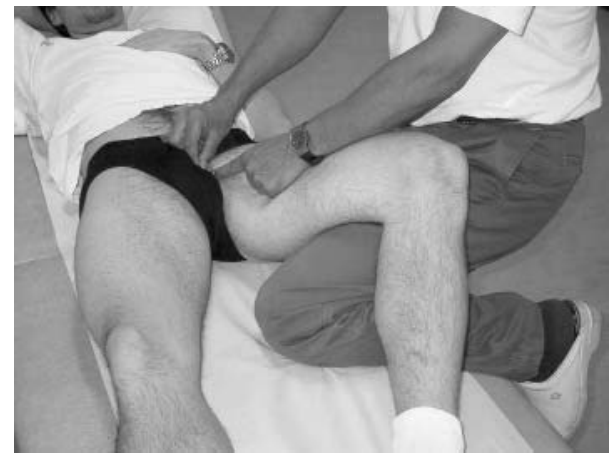

Figure 2 Palpation of the adductor longus muscle insertion.

and the knee slightly flexed. The leg must be relaxed in this position. The subject is instructed to register pain and ignore the natural soreness present in this area. The examiner, using the right hand on the right leg and vice versa, palpates with two fingers the adductor longus tendon and follows the tendon to the insertion at the pubic bone. The insertion area, including the bone, is tested with firm pressure in a radius of about $1 \mathrm{~cm}$.

(A) Pain is recorded as "yes" or "no".

3. Passive stretching of the adductor muscles (fig 3)

The subject lies supine. The examiner abducts the leg to be tested, holding it with one hand and making sure that the foot points straight up. With the other hand, the pelvis is supported to stabilise the testing position. The leg is maximally abducted and the adductor muscles thereby maximally stretched.

(A) Pain in the groin is recorded as "yes" or "no".

4. Palpation of the symphysis joint (fig 4)

The subject lies supine. The symphysis joint is located using gentle palpation with the index finger. The joint is then tested with firm pressure of the index finger tip in the anterior-posterior direction.

(A) Pain is recorded as "yes" or "no".

5. Palpation of the rectus abdominis muscle (fig 5)

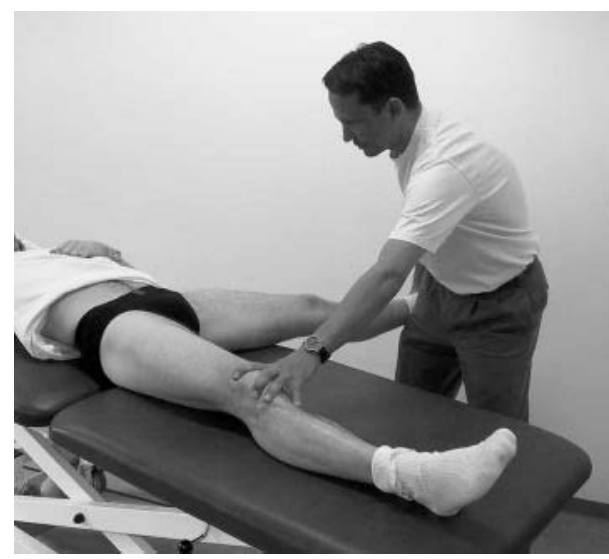

Figure 3 Passive stretching of the adductor muscles. 


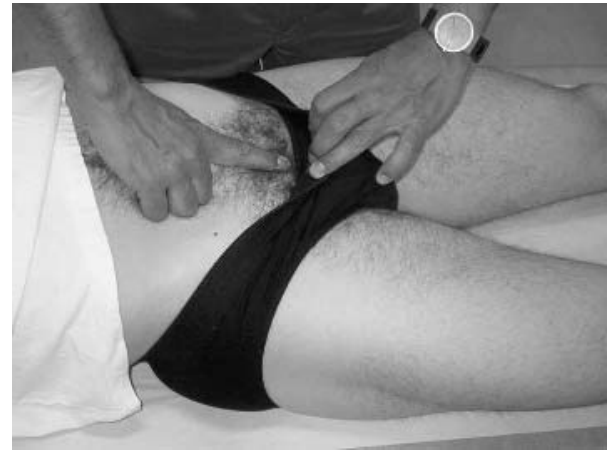

Figure 4 Palpation of the symphysis joint.

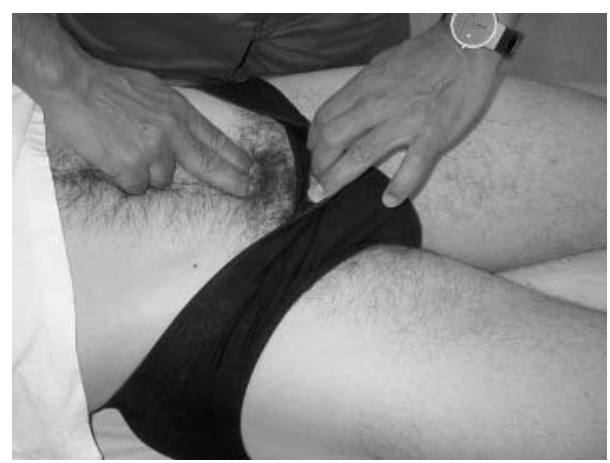

Figure 5 Palpation of the rectus abdominis muscle.

The subject lies supine. The rectus abdominis muscle is palpated at the distal end using two or three fingers. When the insertion at the pubic bone is located, the fingertips are pulled back a few millimetres and then pressed in the posterior direction and then distally down on the pubic bone. The rectus abdominis insertion is thereby palpated firmly.

(A) Pain is recorded as "yes" or "no".

6. Functional testing of the abdominal muscles (fig 6)

The subject lies supine with the hip and knee joints flexed $45^{\circ}$ and the feet against the couch. The arms are folded over the chest. The subject performs a sit up, lifting head and scapulae from the couch. The examiner holds against the subject's knees with one hand and arm and the other arm is pressed against the chest with just enough force to balance the sit up.

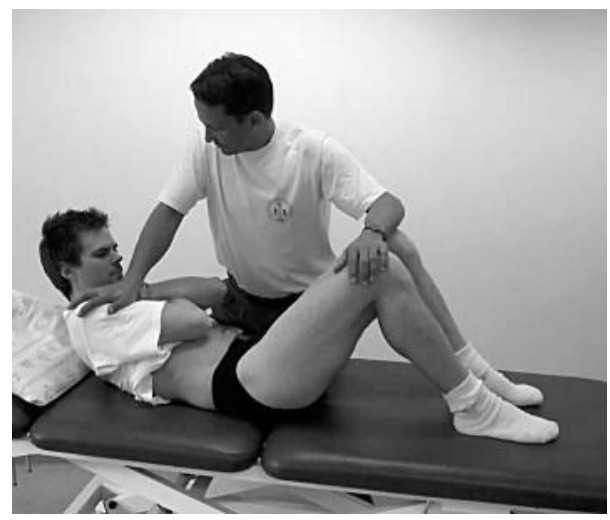

Figure 6 Functional testing of the abdominal muscles.

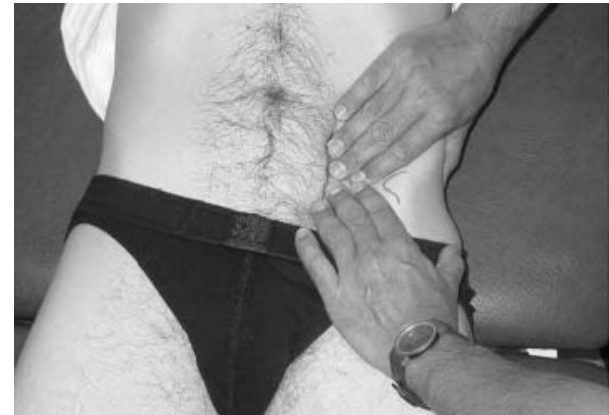

Figure 7 Palpation of the psoas muscle.

(A) Pain in the region of the abdominal muscles is recorded as "yes" or "no".

(B) Strength is evaluated by the examiner as "strong", "intermediate", or "weak".

In the same position the subject performs an oblique sit up, pulling one shoulder towards the opposite knee while the examiner presses against this shoulder.

(C) Pain in the region of the abdominal muscles is recorded as "yes" or "no". The test is performed for both sides.

\section{Palpation of the psoas muscle (fig 7)}

The subject lies supine. The examiner places his/her hands over the lower lateral abdomen at the level of the anterior iliac spine. Palpation is performed with both hands; the fingers should be used to make the palpation as gentle as possible. The lateral edge of the rectus abdominis muscle is located, and palpation is performed on the lateral side of this. The fingers are gently pressed posteriorily while pushing the abdominal structures away to reach the iliopsoas muscle. The subject must be relaxed. When the hands are as "deep" as possible, the subject is told to elevate the foot $10 \mathrm{~cm}$ on the side being tested. The psoas muscle is now palpated firmly over as large an area as possible without lifting the fingers from the skin.

(A) Pain is recorded as "yes" or "no".

8. Functional testing of the iliopsoas muscle (fig 8)

The subject lies supine. The test leg is flexed maximally in the hip and knee joint. The examiner tries to extend the flexed hip by pulling it with one arm wrapped around the femur just proximal to the knee.

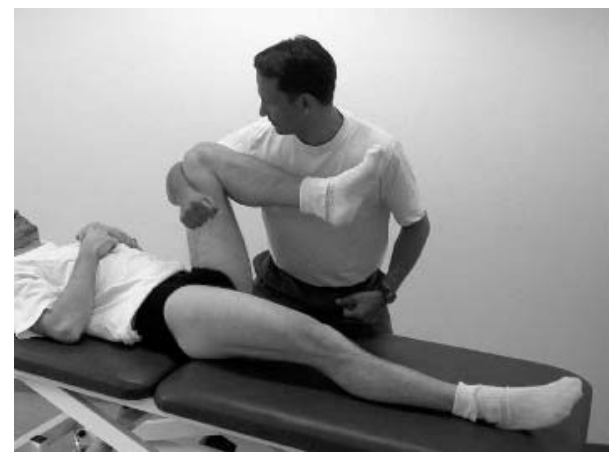

Figure 8 Functional testing of the iliopsoas muscle. 


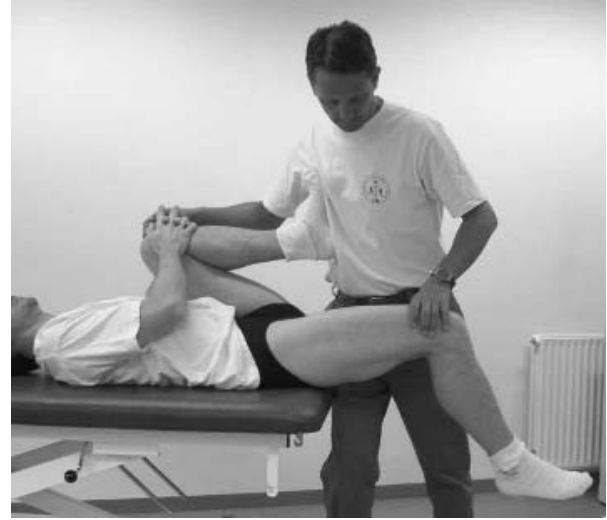

Figure 9 The Thomas test ${ }^{\top}$ (modified) for the iliopsoas.

(A) Pain is recorded as "yes" or "no".

(B) Strength is evaluated by the examiner as "strong", "intermediate", or "weak".

9. The Thomas test ${ }^{7}$ (modified) for the iliopsoas (fig 9)

The subject lies supine with legs hanging from the end of the couch. The subject then flexes one hip by clasping the knee in both hands and pulling it down to his chest. The other leg is hanging relaxed from the end of the couch. At the same time, he lifts his head and shoulders as far as possible. The examiner stands at the end of the couch supporting the position by pressing the side of his/her trunk against the foot of the flexed leg.

(A) The position of the other leg is recorded as "tight" if the femur is elevated above the horizontal level, and "not tight" if it is at or under the horizontal level.

The examiner then places one hand on the femur of the hanging leg just above the knee and presses the leg down to stretch the iliopsoas passively.

(B) When the stretch is felt to be "maximal", pain is recorded as "yes" or "no".

\section{REFERENCES}

1 Renström P, Peterson L. Groin injuries in athletes. Br J Sports Med 1980;14:30-6

2 Hölmich P. Adductor related groin pain in athletes. Sports Medicine and Arthroscopy Review 1998;5:285-91.

3 Nielsen AB, Yde J. Epidemiology and traumatology of injuries in soccer. Am J Sports Med 1989; 17:803-7.

4 Ekstrand J. Soccer injuries and their prevention. PhD thesis, 1982.

5 Ekstrand J, Hilding J. The incidence and differential diagnosis of acute groin pain inmale soccer players. Scand J Med Sports Sci 1999;9:98-103.

6 Hölmich P, Uhrskou P, Ulnits L, et al. Effectiveness of active physical training as treatment for long-standing adductor-related groin pain in athletes: randomised trial. Lancet 1999;353:439-43.

7 Kendall FP, McCreary EK. Lower extremity muscles. In: Kendall FP McCreary EK, eds. Muscles: testing and function. Williams and Wilkins, 1983:158-79.

8 Altman DG. Practical statistics for medical research. London: Chapman \& Hall, 1992.

9 Siegel S, Castellan NJ. Nonparametric statistics for the behavioral sciences. New York: McGraw-Hill, 1988. 